\title{
Assessing dystrophies and other muscle diseases at the nanometer scale by atomic force microscopy
}

\begin{abstract}
Aim: Atomic force microscopy nanoindentation of myofibers was used to assess and quantitatively diagnose muscular dystrophies from human patients. Materials \& methods: Myofibers were probed from fresh or frozen muscle biopsies from human dystrophic patients and healthy volunteers, as well as mice models, and Young's modulus stiffness values were determined. Results: Fibers displaying abnormally low mechanical stability were detected in biopsies from patients affected by 11 distinct muscle diseases, and Young's modulus values were commensurate to the severity of the disease. Abnormal myofiber resistance was also observed from consulting patients whose muscle condition could not be detected or unambiguously diagnosed otherwise. Discussion \& conclusion: This study provides a proof-of-concept that atomic force microscopy yields a quantitative read-out of human muscle function from clinical biopsies, and that it may thereby complement current muscular dystrophy diagnosis.
\end{abstract}

KEYWORDS: atomic force microscopy • diagnosis $\bullet$ muscular dystrophy $\bullet$ nanoindentation

Muscle dystrophies (MDs) are hereditary muscle diseases that cause progressive muscle weakness. MDs often result from various mutated genes that encode distinct musclespecific proteins, with the consequence of a degeneration of myofibers and progressive muscle fibrosis. The most severe forms are Duchenne MD (DMD) and Becker MD (BMD), in which the dystrophin protein is absent or truncated to various degrees. Dystrophin provides a mechanical link between the fiber's inner cytoskeleton and the extracellular matrix. The lack of dystrophin has been associated with more frequent membrane ruptures during muscle contraction, a gradual exhaustion of the muscle stem cells that act to regenerate damaged fibers and eventual muscle fiber loss [1,2]. However, muscle degeneration has been associated with a wider range of clinically and pathologically recognized MDs, and a number of these could be associated with the abnormal function of various other muscle proteins [3-5]. While the loss or the aberrant structure of myofibers can be assessed by histological studies of muscle biopsies, characterization of these MDs often requires the molecular identification of the responsible gene or protein. However, a presymptomatic quantitative functional assay of muscle fibers is currently lacking for such MDs and other similar diseases.

Atomic force microscopy (AFM) was first applied in biology as a surface imaging technique, but has more recently been applied to study the mechanical properties of living cell membranes by nanoindentation [6-8]. The stiffness measured for isolated cultured cells ranges from 0.1 to $100 \mathrm{kPa}$, depending on cell type, sample preparation, indentation depth and calculation model $[6,9]$. The dominance of the cytoskeleton in determining cell stiffness has been shown by different studies. For instance, the depolymerization of the cytoskeleton F-actin by cytochalasin D led to a reduction of fibroblast cell stiffness, showing that the F-actin network plays a crucial role in maintaining the mechanical resistance of the cell membranes [10]. Microtubules may also contribute to cellular stiffness when their depolymerization leads to rearrangement
Ruthger W van Zwieten ${ }^{\ddagger}$, Stefania Puttini ${ }^{\ddagger}$, Małgorzata Lekka, Guillaume Witz, Evelyne Gicquel-Zouida, Isabelle Richard, Johannes A Lobrinus, François Chevalley, Harald Brune, Giovanni Dietler, Andrzej Kulik, Thierry Kuntzer ${ }^{\ddagger}$ \& Nicolas Mermod** *Author for correspondence: Laboratory of Molecular Biotechnology, Center for Biotechnology UNIL-EPFL \& Institute of Biotechnology, University of Lausanne, Station 6, EPFL, 1015 Lausanne, Switzerland Tel.: +41216936151 nicolas.mermod@unil.ch ${ }^{\ddagger}$ Authors contributed equally For a full list of affiliations, please see page 406 
or disassembly of the other filament types. However, most studies involving microtubule depolymerization reported little effect on cell membrane mechanical resistance [11,12].

The pathological loss of the cell membrane's anchorage to the inner cytoskeletal network, or an abnormal constitution of the extracellular matrix or cytoskeleton have also been shown to result in a lower mechanical resistance to subcellular nanoindentation as probed by AFM [13]. For instance, assays of metastatic or nonmetastatic cancer cell lines have revealed a correlation between the ability of the cell membrane to deform at the nanometer scale with its reduced association to the extracellular matrix and ability to invade tissues to form metastasis [14-16].

The AFM approach was also shown to provide a reliable and sensitive method to assay muscle function from fresh muscles explanted from mice at a single muscle fiber resolution [17]. In this assay, the sarcolemmal membrane of individual muscle fibers were probed by nanoindentation using the AFM cantilever tip and the force needed to reach a specific indentation depth was recorded. Assays of dystrophic mouse muscles indicated reduced resistance to deformation compared with healthy muscles, as expected from the lack of the dystrophin-mediated physical link to the cytoskeleton. Dystrophic muscles treated with viral vectors to restore dystrophin expression showed full correction of the resistance to deformation in nearly all of the muscle fibers. This indicated that AFM truly probed the myofiber's elastic properties as determined by dystrophin rather than later muscle degeneration events, and that it can provide a quantitative assessment of the efficacy of gene-based therapeutic approaches and muscle function in animal models.

In this study, the authors wished to determine if AFM may be applied to human frozen biopsies as available at clinical diagnostic centers, and whether it can be used to detect alterations of myofiber resistance in the context of various other human muscular dystrophies and myopathies. The findings from this study indicate that the AFM approach is a sensitive method to assess human muscles, and that biopsies from DMD and BMD patients can be distinguished from those of matched healthy individuals. Interestingly, AFM also allowed the detection of muscle fiber anomalies in biopsies corresponding to various other muscle diseases, including from patients whose muscle weaknesses could not be attributed to a known molecular or genetic defect. Overall, the authors conclude that AFM may provide a useful method to complement current diagnosis tools of known or uncharacterized human muscle diseases, in research and in a clinical context.

\section{Materials \& methods}

Biopsy preparations

This study was reviewed and approved by the ethical institutional review board. All muscle biopsies from diagnosed and consulting patients had been previously isolated and frozen for routine diagnosis purposes at the Lausanne University Hospital, Switzerland, except for those from DMD patients and control paravertebral muscle biopsies, which were collected and kindly provided by the Myology Institute in Paris, France. Muscle biopsies from three DMD boys (14, 14 and 15 years old) and two healthy boys (11 and 16 years old) were available. Biopsies had been snap frozen in liquid nitrogen or in liquid nitrogen-cooled isopentane, as performed for molecular analysis or histology purposes, respectively. Biopsies from healthy control patients undergoing hip prosthesis surgery were obtained under informed consent. Healthy patient biopsies were divided into three equal samples of approximately $0.7 \mathrm{~cm}^{3}$, a size comparable to that of surgical biopsies obtained from dystrophic patients. One sample was placed in DMEM and kept on ice directly following the surgical removal, and the elastic properties of individual myofibers were probed using AFM within $8 \mathrm{~h}$ of surgical removal without a freezing procedure. Other human biopsies were frozen either by direct immersion in liquid nitrogen or in liquid nitrogen-cooled isopentane, as performed in clinical institutions. Mouse muscles were frozen by cooling at $-80^{\circ} \mathrm{C}$ after immersion in DMEM supplemented with $10 \%$ dimethylsulfoxide. Frozen samples were stored in liquid nitrogen until being thawed and processed for AFM assays.

\section{AFM assays}

Where applicable, frozen human muscle biopsies were allowed to thaw at room temperature for $1 \mathrm{~h}$ before processing for the AFM assay. Human muscle sectioning was performed parallel to the myofibers in a rat brain slicer matrix (Plastics One, VA, USA) with a spacer width of $1 \mathrm{~mm}$ to yield slices of uniform thickness. In order to preserve myofiber integrity, muscle slices were immediately placed in DMEM and chilled on ice until they were measured by AFM. Muscle slices were immobilized with muscle fibers lying flat on the AFM sample support (Figure 1A). Mouse tibialis anterior (TA) samples were prepared similarly, as previously described [17].

The AFM measurements were performed using a device XE-120 (Park Systems, Suwon, Korea) equipped with the 'liquid cell' setup. The uncoated silicon nitride cantilevers (MLCT-AUHW, Veeco, NY, USA) were characterized by a tip radius of $50 \mathrm{~nm}$, open angle of $35^{\circ}$ and nominal cantilever spring constant of $0.01 \mathrm{~N} / \mathrm{m}$. The force curves - that is, the dependence between the 


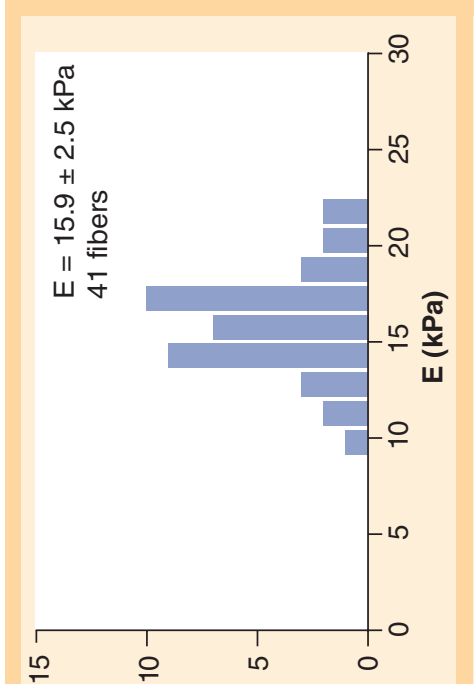

(C) (u) sıəq!y

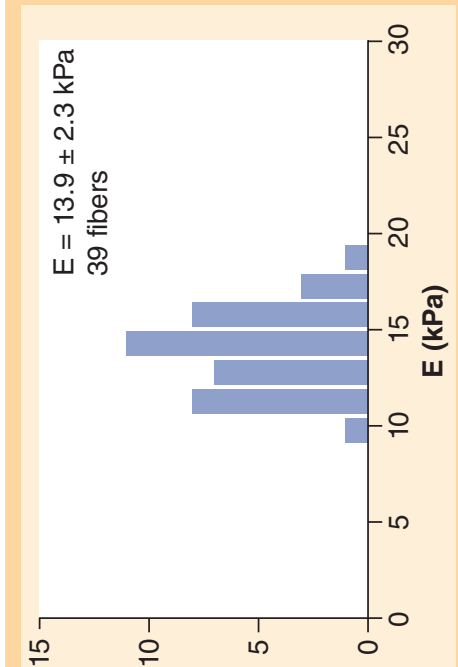

(D) (u) sıəq!」

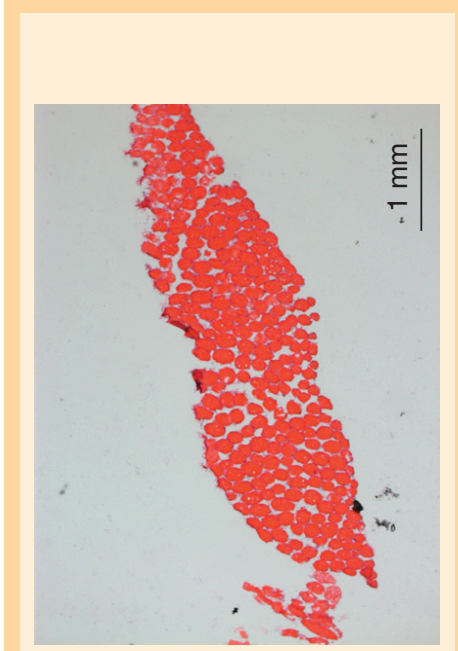

(๔)

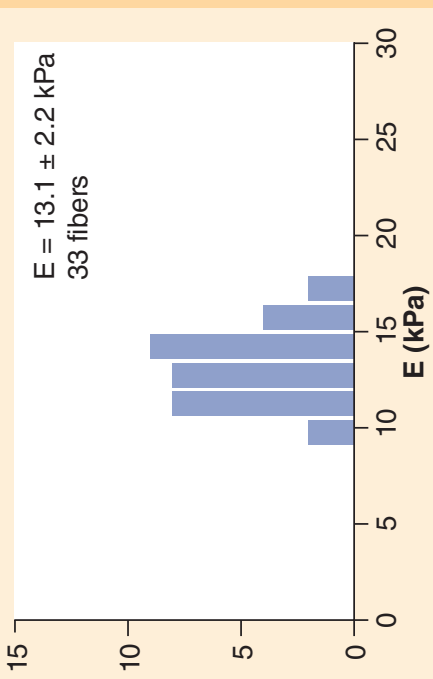

(4)

(u) s.ıəq!

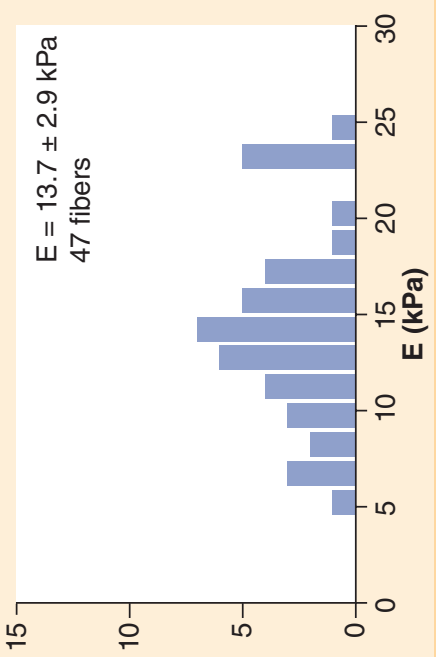

(1) (u) s.əq!」

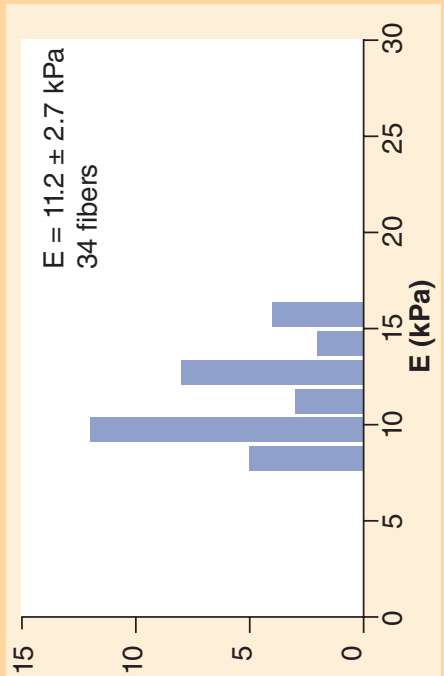

(a) (u) sıəq!y

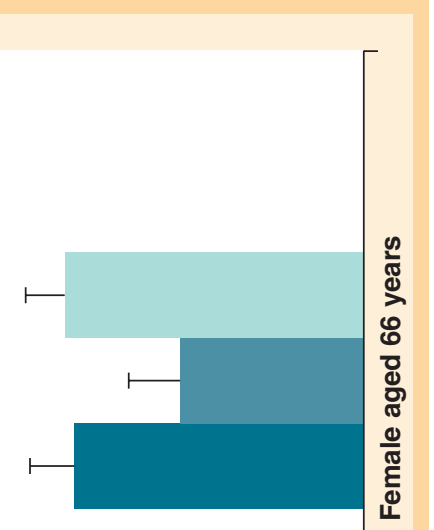

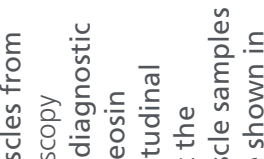

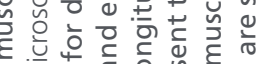

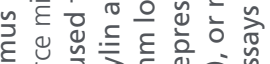
है

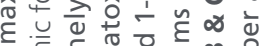
气

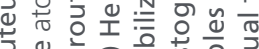
วัँ

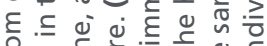

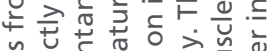
๘

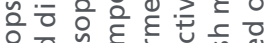

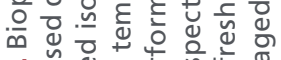

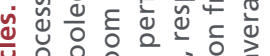

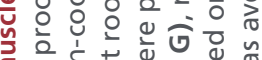

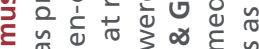

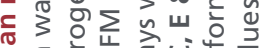

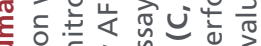

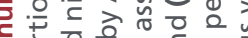

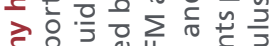

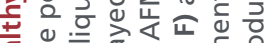
ब 范过 రั

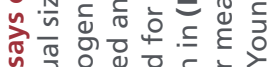

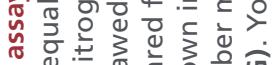
สั

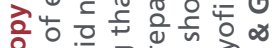

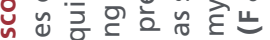

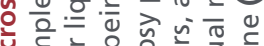

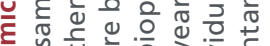
ఖ

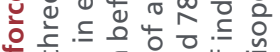

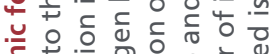

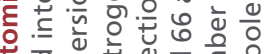
tั0 ठ 등 은 은 은 는

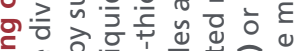

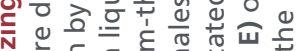
ఖँ

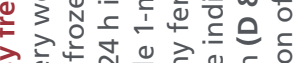

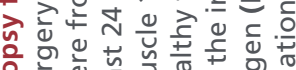

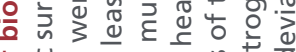

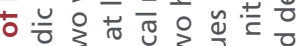

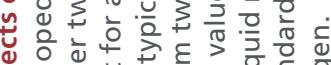

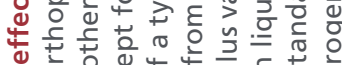
ब 이일

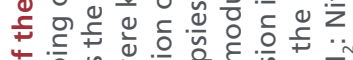

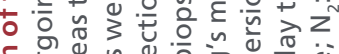
등 원

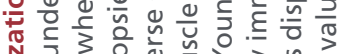

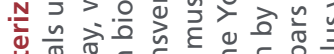

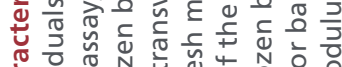
蛋:

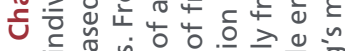

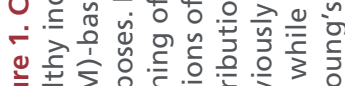

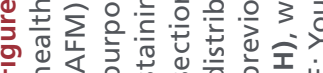


cantilever deflection (proportional to the load force) and the distance between the probing tip and sample position were recorded at the approach speed of $7 \mu \mathrm{m} / \mathrm{s}$ and the Z-range equaled $10 \mu \mathrm{m}$. Each tissue section from a given patient was divided into two slices. For each measured tissue slice, $15-20$ force maps $(4 \times 4)$ were acquired from randomly chosen areas over the whole tissue section. Thus, for a given human sample, measurements were repeated twice, which resulted in 480-640 recorded curves. The Young's modulus calculations were performed as previously described for murine muscles [17]. Briefly, the force-versus-indentation curves were determined by subtracting the calibration curve (recorded on a glass coverslip) from the curve recorded for the studied muscle sample. To this curve, the Hertz model was fit by assuming the conical shape of the probing tip. The fit was performed using the proprietary code 'Force' developed at the Institute of Nuclear Physics PAN, Kraków, Poland. In this program, the contact point is defined as the point where the deflection of the cantilever is higher than the level of the base line in force curve, as determined manually. The resulting individual Young's moduli were used to calculate the final modulus values. Final Young's modulus values were obtained by averaging the Young's modulus measurements, as obtained from individual muscle fibers pertaining to a given muscle type and biopsy storage condition. Statistical evaluation was performed on individual indentation measurements using the unpaired student's $t$-test with unequal variance, and where applicable, mean values and standard errors are shown. Table 1 \& Figures 1 \& 2 display mean values plus the standard deviation.

\section{Cytochalasin D treatment of muscle biopsies}

Muscle slices as prepared for AFM measurement were incubated in DMEM supplemented with $0.1125 \mu \mathrm{m}$ cytochalasin $\mathrm{D}$ at $37^{\circ} \mathrm{C}$ for $30 \mathrm{~min}$. Afterwards, slices were rinsed in $1 \mathrm{ml}$ DMEM before AFM measurement.

\section{Histology}

Dystrophin staining was performed on $10-12 \mu \mathrm{m}$ frozen sections that were incubated with antidystrophin antibodies (NCL-Dysl, diluted 1:2; Novocastra-Leica Microsystems, Wetzlar, Germany) overnight at $4^{\circ} \mathrm{C}$. Afterwards, sections were incubated for $35 \mathrm{~min}$ at room temperature with an antimouse secondary antibody conjugated with a horseradish peroxidase-labelled polymer $\left(\right.$ EnVision $^{\circledR}+$ System, Dako, Baar, Switzerland). Subsequently, sections were incubated for $3 \mathrm{~min}$ with 3,3'-diaminobenzidine (EnVision+ System), so as to provide a brown color to dystrophin at muscle fiber membranes. Finally, sections were counterstained with Mayer's hematoxylin and eosin, and they were mounted with Eukitt (Sigma-Aldrich, MO, USA), so as to stain muscle fiber nuclei in light blue and the cytoplasm in light pink, in order to reveal the muscle fibers.

\section{Results}

Establishment of an AFM assay of human muscle biopsies

Earlier AFM studies had suggested that elasticity-based nanoindentation assays are capable of detecting changes in the stiffness of myofibers' sarcolemmal membranes, as elicited by the presence or absence of dystrophin in freshly isolated mouse TA muscles [17]. Here, the authors first sought to investigate the feasibility of applying AFM to previously frozen clinical muscle biopsies, as may be obtained from human patients. The authors first assessed gluteus maximus muscle biopsies that were obtained from healthy human donors, where part of the biopsy was used fresh, while other portions were submitted to a prior freezing procedure. Samples were then thawed and sectioned longitudinally to preserve myofiber integrity to yield sections with a thickness corresponding to approximately ten myofibers (Figure 1A). These slices were laid and immobilized on a solid support to perform nanoindentation AFM assays, and the Young's modulus values representing the muscle fiber resistance to elastic deformations were determined, as described earlier [17].

The mechanical resistance of fresh muscles varied from 7 to $16 \mathrm{kPa}$ for distinct healthy humans (Supplementary Figure S1; see online at www.futuremedicine.com/doi/suppl/10.2217/nnm.12.215), and thus variability was larger than that determined previously with fresh muscles from the inbred, and thus genetically homogeneous, C57Bl/6j mice [17]. Differences in human muscle resistance values could not be strictly correlated with age or gender of the human volunteers. The muscle biopsy portions that had been frozen in cooled isopentane were well preserved, as they yielded Young's modulus histograms that were nearly identical to those obtained from freshly measured muscles (Figures 1B \& $1 \mathrm{C}$ vs $1 \mathrm{~F} \&$ 1G). Some of the Young's modulus values were somewhat lower after freezing by direct immersion in liquid nitrogen (Figures 1D \& 1E). However, these samples appeared to be sufficiently well preserved for AFM analysis as they yielded average Young's modulus values that were within a normal range of variability, as obtained from fresh muscle biopsies from seven healthy individuals (Figure 1H \& Supplementary Figure S1).

When assessed similarly, TA muscles from healthy mice yielded comparable AFM profiles when comparing fresh and frozen mouse muscles (Supplementary Figures S2A \& S2B). Frozen muscles from dystrophic (mdx) mice yielded lower Young's modulus values (Supplementary Figure S2D), as reported previ- 


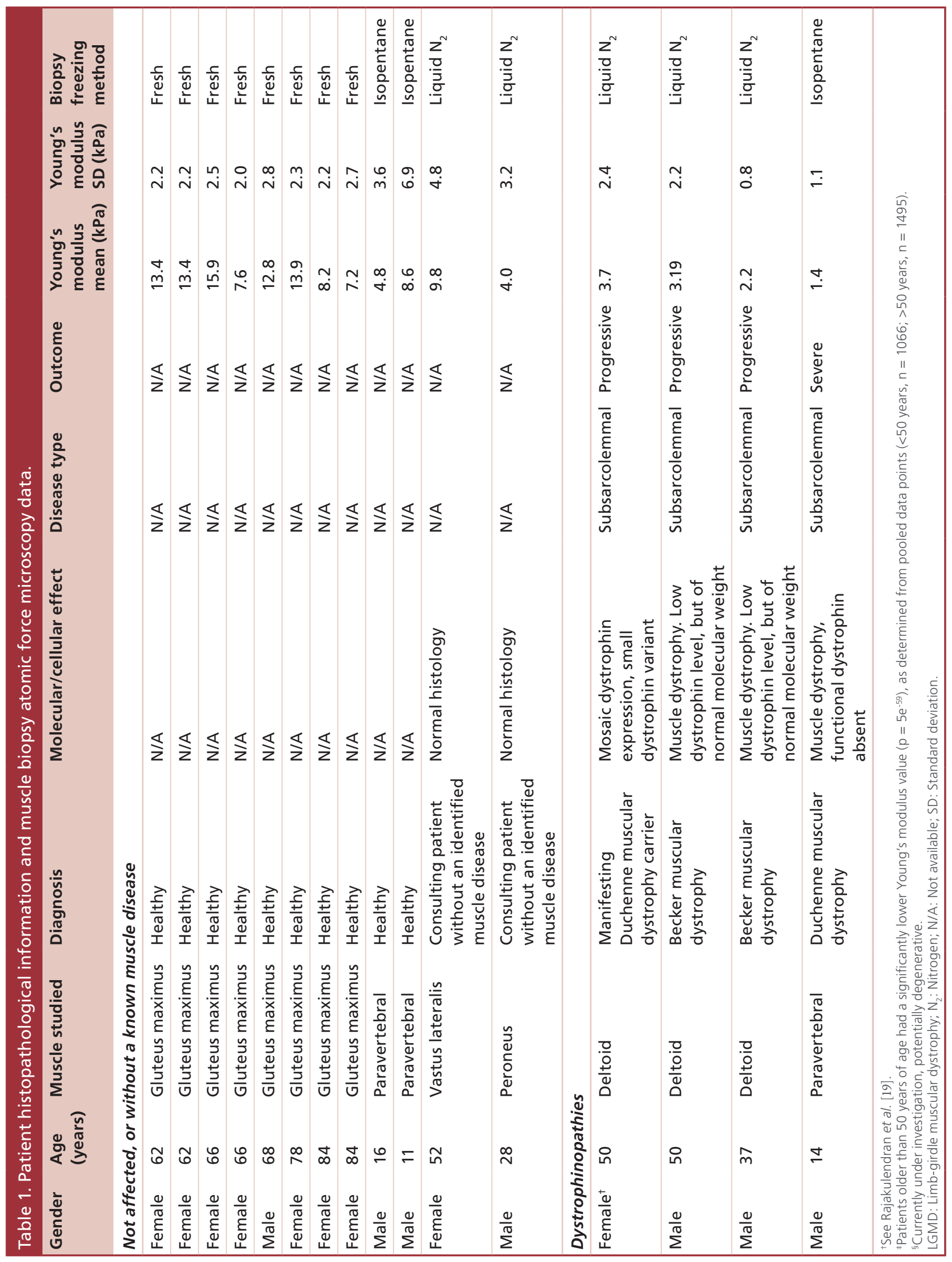




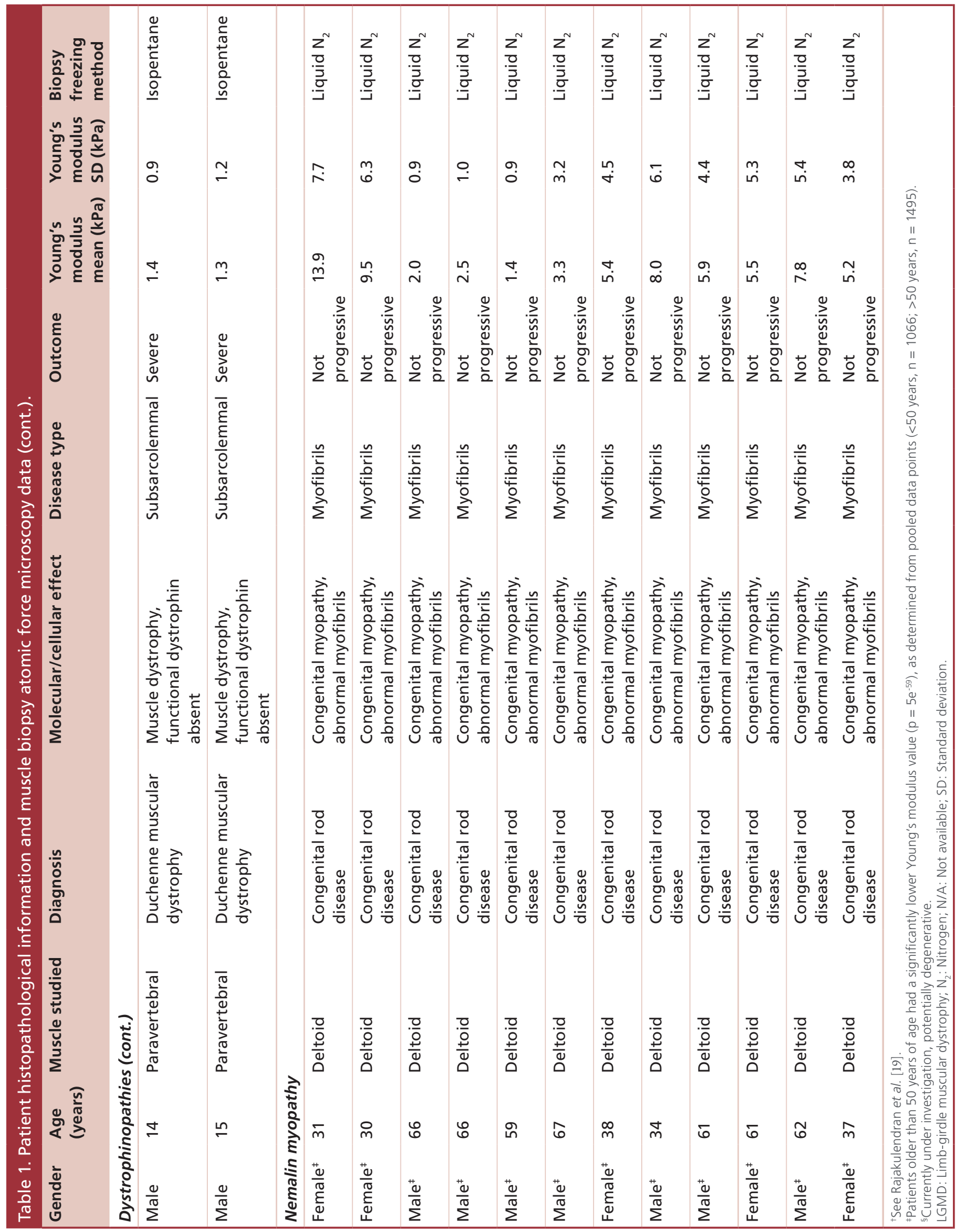




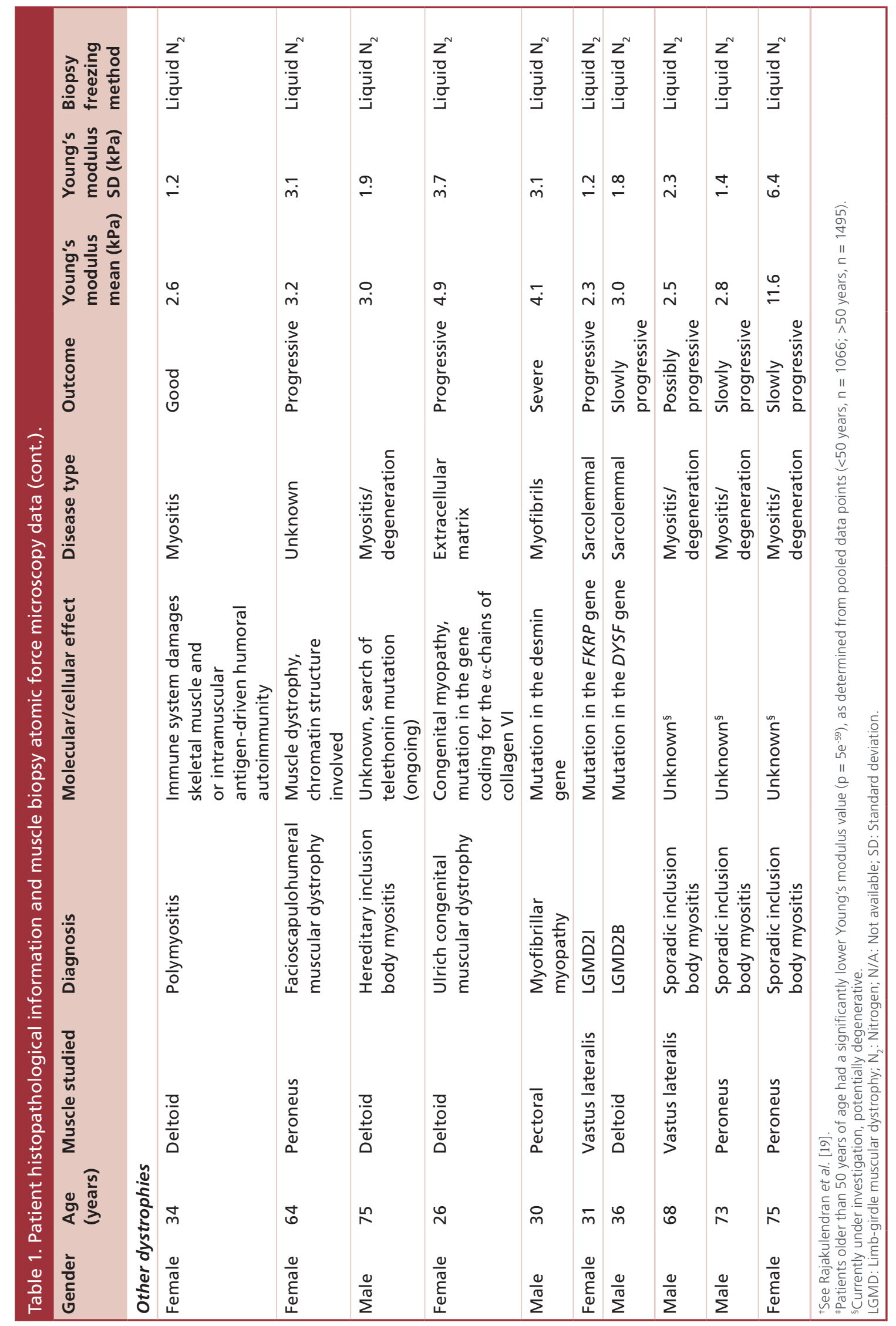


ously from fresh murine muscles [17], confirming that accurate AFM profiles can be obtained from frozen muscle explants. Interestingly, the bulk of healthy murine myofibers yielded Young's modulus values in a $2-15 \mathrm{kPa}$ range, whereas a minority of more resistant fibers was also observed, potentially reflecting different fiber types (Supplementary Figures S2A \& S2B). This contrasted the relatively stiffer and more homogeneous 10-25 kPa range observed for fresh biopsies from aged healthy humans (Figures 1B \& 1C).

\section{AFM assays of muscles from DMD patients}

The authors next proceeded to determine whether frozen biopsies from dystrophic patients could be distinguished from those of healthy individuals. To this end, liquid nitrogen-cooled isopentane frozen paravertebral muscle biopsies from DMD and healthy boys were assessed by AFM and the Young's modulus values were recorded (see Table 1 for all values). Assays of paravertebral muscle biopsies from healthy boys yielded a range of Young's modulus values that were indicative of resistant muscles (Figures 3A \& 3B), while DMD human muscle fibers had much lower resistance to deformation (Figures 3C-3E). Muscles from DMD patients displayed significantly lower average resistance values than those of healthy young subjects $(1.10 \pm 0.04 \mathrm{kPa}$ vs $3.50 \pm 0.10 \mathrm{kPa}$, respectively; $\left.\mathrm{p}=3.6 \times 10^{-73}\right)$, underlining the discriminative resolution of AFM. Representative immunohistological staining of muscle biopsies is shown for a healthy and DMD patient in Figures 3F \& 3G, respectively, confirming the lack of dystrophin in the diseased muscle. Interestingly, the Young's modulus values determined for healthy boys were rather heterogeneous (Figures 3A \& 3B), with at least two populations of muscle resistance fractions, as obtained from young healthy mice (Supplementary Figure S2B). Furthermore, muscles from young humans and mice displayed quite similar elasticity values. This contrasted with the more homogenous profile and on average stiffer properties yielded by muscles of older healthy patients (Figures 1B \& 1C). The difference correlates with age rather than with muscle function or species, as healthy human gluteus maximus and healthy murine TA are both leg-associated muscles that contribute to posture maintenance, but they yielded distinctive homogeneous or heterogeneous profiles depending on the donor age.

Although the absolute Young's modulus values can differ when comparing young and aged healthy humans, resistance values were similarly very low and yielded comparable homogeneous profiles when assessing dystrophic muscles from human or murine origin (Figure 2 \& Supplementary figure S2B), pro- viding a unambiguous AFM profile for the dystrophic muscles. These results taken together imply that the AFM assay of muscle function may be performed on frozen human muscle biopsies, and that it may provide a clinically significant evaluation of dystrophinopathies.

\section{AFM values of biopsies representing various muscle disorders}

To further assess whether AFM may provide a functional evaluation of human muscles, biopsies from patients affected by other types of dystrophies that result from mutations in various components of the dystroglycan complex were next investigated. BMD is a dystrophinopathy caused by a truncated but partly functional dystrophin protein, yielding a milder muscle dysfunction than DMD and at a later age. The fukutin-related protein mutated in limb-girdle MD (LGMD)2I may control the glycosylation status of dystroglycan, ensuring its association to proteins of the extracellular matrix in healthy subjects [18]. The authors therefore hypothesized that both types of mutations may affect the stability of the dystroglycan complex and/or its ability to link the extracellular matrix with the cytoskeleton, thereby leading to MD in vivo and possibly also to an altered AFM assay profile.

Biopsies from BMD patients yielded low mechanical resistance when compared with a healthy male (Figures 2A \& 2B). The Young's modulus values of the BMD patients did not depend on age, as abnormally low values were noted for both a middle-aged and older patient (Table 1). Furthermore, the resistance values of BMD muscles, although very low, were not as low as those obtained from muscles from patients affected by DMD $(1.40 \pm 0.001 \mathrm{kPa}$ vs $2.90 \pm 0.006 \mathrm{kPa}$, respectively; $\left.\mathrm{p}=8.3 \times 10^{-36}\right)$, thus correlating well with the more severe muscle dysfunction associated to the latter disease. Furthermore, the 37-year-old patient with the earlier onset and more severe form of BMD had a lower Young's modulus value than the 50-year-old BMD patient, indicating that the elasticity value relates to the severity of these dystrophinopathies. This finding was further confirmed by AFM of a biopsy from a rare female manifesting the DMD carrier [19]. Its biopsy yielded a mean Young's modulus value that was higher than those found in BMD males, but below the range found for healthy subjects, further supporting the conclusion that myofiber elasticity correlates with dystrophin function in humans.

Likewise, the biopsy from a LGMD2I female patient showed anomalously low Young's modulus values compared with a healthy female (Figures 2C \& 2D). This paralleled an assay performed on a murine model for 

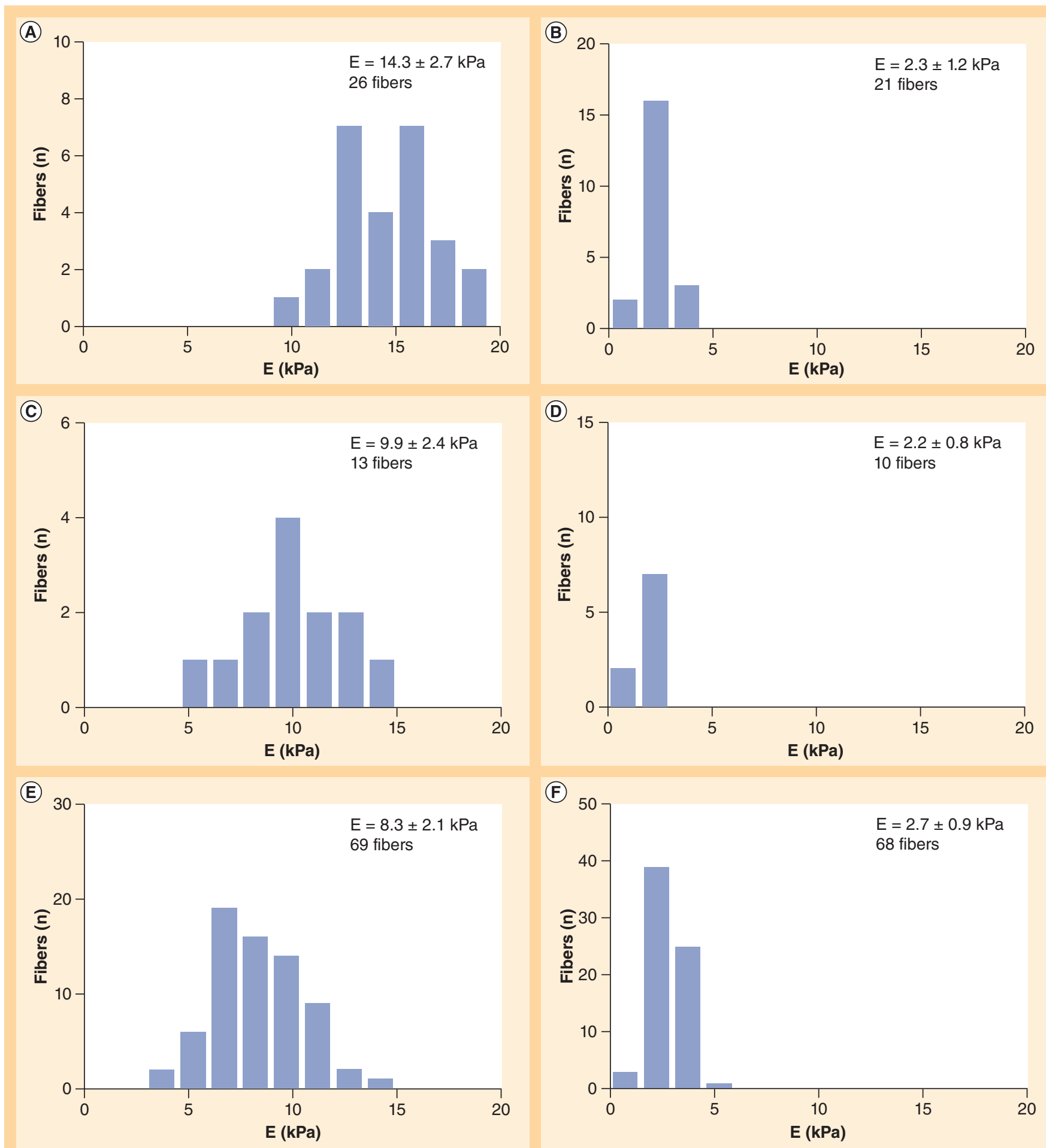

Figure 2. Muscle biopsies from Becker muscle dystrophy and limb-girdle muscular dystrophy type 21 patients and mice models yield a dystrophic atomic force microscopy assay profile. Human biopsies previously frozen in liquid nitrogen were thawed and processed for the atomic force microscopy assays alongside freshly explanted mouse muscle. Young's modulus elasticity profiles are shown for biopsies of a (A) gluteus maximus muscle of a 68-year-old healthy human male subject; (B) a deltoid muscle from a 37-year-old male Becker muscle dystrophy patient; (C) a healthy gluteus maximus muscle taken from a 78-year-old female human subject and for a (D) vastus lateralis muscle from a 31-year-old female limb-girdle muscular dystrophy type 21 patient. Young's modulus profiles were also determined on a (E) tibialis anterior muscle from a healthy mouse control aged 5 weeks and from a (F) tibialis anterior muscle obtained from a FKRP knock-out mouse as a model of limb-girdle muscular dystrophy type 2I, also 5 weeks old and from the same littermate as the animal analyzed in (E).

E: Young's modulus value. 

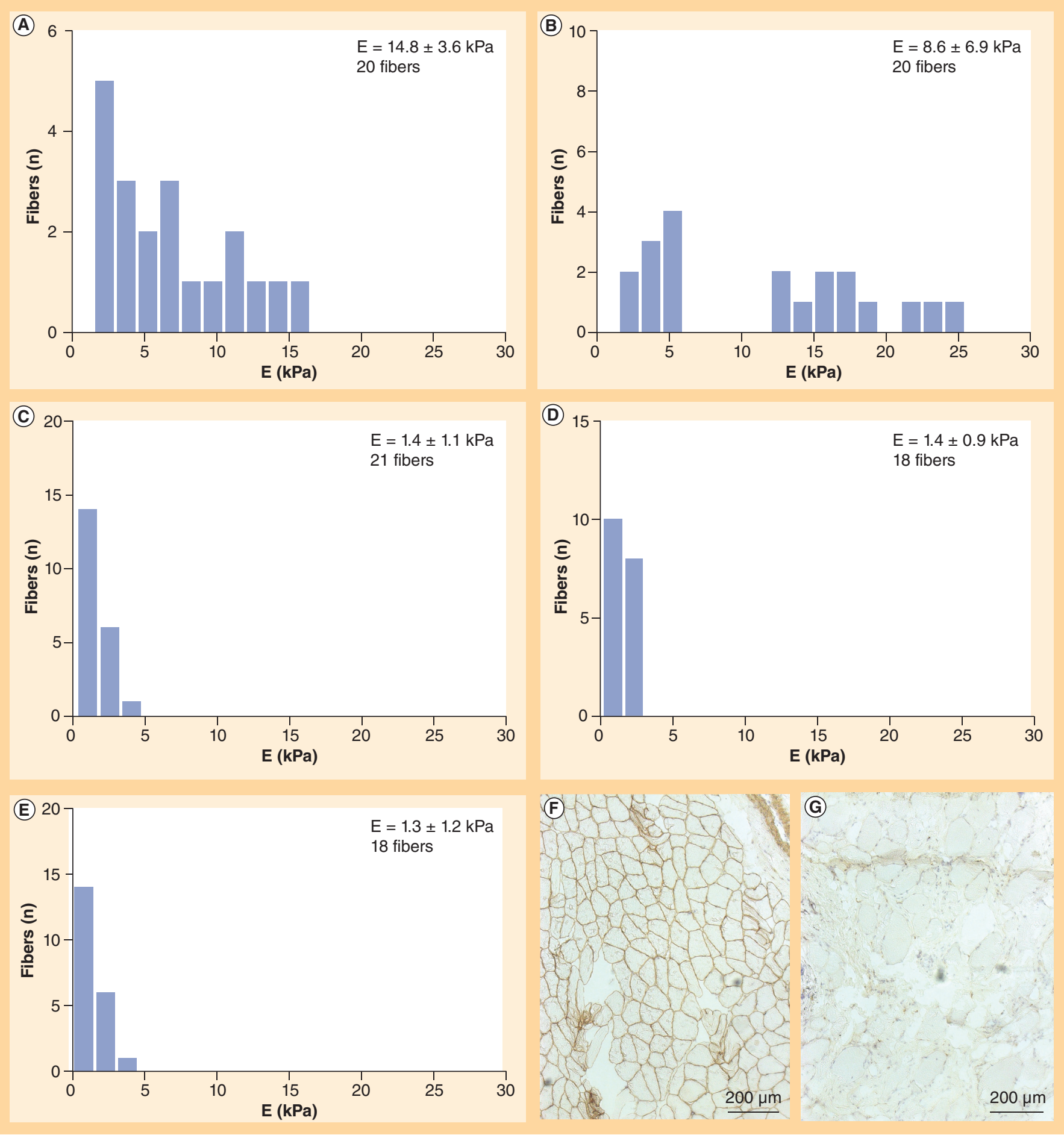

Figure 3. Duchenne muscular dystrophy biopsies have a distinctly low resistance to deformation. Paravertebral muscle biopsies of $0.5 \mathrm{~cm}^{3}$, previously frozen in liquid nitrogen-cooled isopentane, were thawed and processed for atomic force microscopy assays.

(A) Healthy male aged 16 years. (B) Healthy male aged 11 years. (C) Duchenne muscular dystrophy male aged 14 years. (D) Duchenne muscular dystrophy male aged 14 years. (E) Duchenne muscular dystrophy male aged 15 years. Dystrophin immunohistological staining was performed on sections of the muscles of a (F) healthy and (G) dystrophic patient.

E: Young's modulus value.

LGMD2I bearing a homozygous knock-in of a human mutation in the gene encoding the fukutin-related protein (Figures 2E \& 2F) and those obtained from a human patient affected by a LGMD2B muscle disease (Table 1). Again, the Young's modulus values were low, but not as low as those from patients affected by the more severe 
$\mathrm{DMD}$, indicating again that the AFM-determined resistance values may provide a quantitative assessment of the severity of muscle dysfunction.

To strengthen this conclusion, the authors next expanded their study to a larger collection of available frozen human muscle biopsies. Overall, muscles from patients affected by 11 diseases were probed (Table 1). All assayed muscles from patients with dystrophinopathies had extremely low mechanical resistance, with no fiber having normal or near-normal AFM-assessed elasticity values. Patients of various ages and genders affected by various other types of muscle disorders with weakness, including polymyositis, hereditary inclusion body myositis, Ulrich congenital MD and myofibrillar myopathy, all yielded various degrees of abnormal muscle resistance as detected by the AFM assay (Table 1).

Having determined that muscle dysfunction associated with a variety of previously diagnosed muscle diseases can be detected by the AFM assay, the authors turned to muscle diseases where diagnosis may be more difficult. For instance, the authors assessed a family affected by a novel type of nemaline myopathy for which no molecular or genetic marker is known, whereas histology or other available assays do not allow unambiguous diagnosis [20]. Most, if not all, of the family members appear to be affected by a mild but variable muscle weakness, which is difficult to diagnose as muscle performance can be also affected by the lifestyle and training particular to each individual. Distinct family members yielded different AFM profiles, with some individuals yielding AFM profiles close to those of BMD patients, whereas others yielded normal or near-normal muscle resistance (see Supplementary Figures S3A \& S3B for representative AFM profiles). Older family members had muscles of significantly lower resistance $(3.90 \pm 0.01 \mathrm{kPa}$ vs 7.70 $\pm 0.01 \mathrm{kPa} ; \mathrm{p}=5.0 \times 10^{-59}$, when comparing individuals older or younger than 50 years, respectively), while gender or fiber-type composition did not affect the AFM Young's modulus values (Table 1 and data not shown). Interestingly, incubation of the nemaline myopathy biopsy sections with cytochalasin $\mathrm{D}$ revealed fibers that were resistant to the softening induced by this treatment (Supplementary Figure S4). This indicated that a portion of the fibers had a stiff behavior that was independent of the cytoskeleton integrity, potentially reflecting the presence of proteinaceous aggregates, as seen by microscopy in some patients. In addition, the heterogeneity seen in the AFM profiles from different affected nemaline myopathy family members was also found in sporadic inclusion body myositis (Supplementary Figures S3C \& S3D), possibly reflecting similar molecular depositions.

Finally, the authors also assessed two patients who were consulting for suspected muscle weakness but were classified as healthy, as muscle biopsy histology did not reveal abnormal structures and no known muscle disease diagnostic marker was found. For one of the two patients, a 30-year-old male, a low Young's modulus value was found, similarly to what was encountered in patients displaying known MDs (Supplementary Figure S5). Overall, the authors conclude that probing muscle biopsies by AFM indentation at the nanometric scale may be capable of revealing mild muscular defects, and this even in the absence of fully developed or otherwise detectable symptoms.

\section{Discussion \& conclusion}

This study reports a first in situ functional assessment of a variety of human muscle fibers by AFM. When comparing fresh human muscle samples with frozen biopsies obtained from clinical collections, we concluded that valid measurements can be obtained from biopsies as routinely processed and stored at hospitals, and that this approach can be applied to distinguish healthy from diseased human muscles. Both the Young's modulus histogram profile and the mean modulus value are well preserved in frozen tissues, whether from human or mice origin. Although several freezing methods were found to be applicable, we recommend using biopsies frozen in liquid nitrogen-cooled isopentane for future AFM studies of human muscles, as this approach did not alter myofiber resistance to any detectable extent.

We previously reported that $\mathrm{mdx}$ mice lacking dystrophin have low resistance myofibers compared with those of healthy mice, and that dystrophin expression by a gene therapy approach did restore a normal AFM profile from the animal dystrophy model [17]. This suggested that the dystrophin-mediated attachment of the sarcolemmal membrane to the underlying actin cytoskeleton was providing resistance to the mechanical indentation induced by the AFM cantilever pressure. This report shows that fibers of abnormally low resistance occur more generally in conjunction with various other muscle diseases, as characterized by a number of distinct molecular or cellular defects. These ranged from the disjunction of the dystroglycan complex connection to the myofiber's actin cytoskeleton, as in DMD or BMD patients, or to the extracellular matrix as in fukutin-related protein, to muscle diseases associated to an immune and inflammatory response of the muscle tissues, as in polymyositis. All of these disease share the common feature of muscle strength loss. The extent of muscle strength loss associated with particular diseases correlated well with the loss of muscle resistance to elastic deformation, as probed by AFM. This correlation is further supported by the comparison of biopsies from patients affected by similar diseases, but with a different severity. For instance, 
DMD patients who fully lack dystrophin have lower Young's modulus values than those obtained from the BMD patients assessed in this study that express abnormally low but detectable levels of full-length dystrophin (Table 1). Furthermore, the 37-year-old BMD patient showed a dystrophic AFM profile that was more severe than the profile of the BMD patient aged 50 years, which correlated well with the earlier and more severe phenotype of the 37-year-old patient. A number of other muscle diseases that have later disease onset and/or severity consistently yielded AFM values that were between those of the most severely affected DMD patients and those obtained from healthy volunteers. We therefore conclude that use of the AFM assay is not limited to the detection of diseases that affect the sarcolemmal membrane resistance to elastic deformation, but that it probes a more generally relevant biophysical property of the muscle that underlies strength.

The AFM indentation assay probes the resistance of inner cellular structures at a nanometric scale, as defined by the depth at which the pressure required to indent the membrane is recorded [17]. In this study, this was assessed at a depth of approximately $500 \mathrm{~nm}$ after the initial contact point between the approaching cantilever tip and the myofiber surface. As this depth extends beyond the $10 \mathrm{~nm}$ sarcolemma and $150 \mathrm{~nm}$ dystrophin link, the sarcolemmal membrane is pushed further within the myofiber until it reaches a more resistant inner structure, deeper than the actin microfilaments directly underlying the sarcolemma. In some cases, the cantilever might probe rigid structures that result from particular muscle pathological processes. For instance, in the sporadic inclusion body myositis, particular patients can show rod-shaped proteinaceous structures deposited within some of the myofibers. Interestingly, heterogeneous resistance values were observed in the myofibers of one such female subject, where some fiber locations were found to correspond to stiffer structures than those of the surrounding fibers in the biopsy, or those from healthy subject fibers.

Alternatively, the inner structures probed by the cantilever might be the contractile apparatus that mediates muscle strength. This interpretation is supported by the correlation of muscle strength loss, as elicited by the various diseases assessed, with the Young's modulus value that was determined from the myofibers. Thus, the ability of the probed cellular structures to resist deformation, as exerted by the cantilever tip pressure, might be directly correlated to the functional resistance of the sarcomeric contractile unit. In future studies, it will be interesting to assess this possibility further by probing muscles with impaired components of the sarcomeric structure, such as muscles lacking titin, the protein that acts as a molecu- lar spring that connects sarcomeres to provide the passive elasticity of the muscle. While the main aim of this study was to establish the elastic properties of tissue sections as a potential diagnostic nanometric marker, another interesting perspective will be to perform AFM-based imaging to determine if various types of diseases may be distinguished on the basis of possibly distinct morphological changes. In any case, although the precise structure probed by AFM remains to be identified, we conclude that the AFM-mediated nanoindentation assay may be capable of probing muscle function, as affected by a wide range of disease associated with muscle weakness.

Interestingly, we detected very soft fibers in one consulting patient rated as having an undiagnosed muscle condition, as no objective criterion justifying a muscle disease diagnosis could be identified by other assays, including skeletal muscle strength, enzyme levels, electrodiagnostic study, muscle imaging and histology, which were all found to be within the normal variation limits. The abnormal AFM profile may thus point to an underlying muscle disease not diagnosed as yet by other approaches. Biopsies from the members of a family affected by another muscle condition that is difficult to diagnose by conventional means, having a mild form of atypical nemalin myopathy, were also found to contain soft fibers, although in varying proportions. Thus, AFM may provide a useful additional approach to diagnose MDs, even in the absence of a severe phenotype or of a known molecular disease mechanism. Overall, we conclude that AFM shows promise as an additional diagnostic tool in routine clinical use and possibly also to evaluate clinical trial outcome.

\section{Future perspective}

We speculate that AFM will be increasingly applied to the preclinical and clinical evaluation of muscle therapies owing to its high sensitivity and ability to give a functional read-out from single fibers embedded within the muscular tissue. The high-resolution data that can be generated by this novel approach may also aid in the optimization of gene therapy vector dosage, administration route and regimen, by improving the outcome of preclinical and clinical trials. In the long-term, AFM may become a standard diagnosis tool for myopathies, facilitating the selection of the most effective clinical treatment strategy.

\section{Acknowledgements}

The authors thank S Gros-Spina and S Trepey for help and dedicated management of muscle biopsies. The authors also acknowledge T Voit, S Vasseur and M Chapart-Leclert from the Institut of Myologie and MYOBANK (Paris, France) for giving the authors Duchenne muscle dystrophy muscle biopsies. 
Financial \& competing interests disclosure

This work was supported by the Swiss Foundation for Research on Muscle Diseases to N Mermod, and by an interdisciplinary research grant from the faculty of Biology and Medicine of the University of Lausanne to T Kuntzer, N Mermod and A Kulik. The authors have no other relevant affiliations or financial involvement with any organization or entity with a financial interest in or financial conflict with the subject matter or materials discussed in the manuscript apart from those disclosed.

No writing assistance was utilized in the production of this manuscript.

\section{Ethical conduct of research}

The authors state that they have obtained appropriate institutional review board approval or have followed the principles outlined in the Declaration of Helsinki for all human or animal experimental investigations. In addition, for investigations involving human subjects, informed consent has been obtained from the participants involved.

\section{Open access}

This work is licensed under the Creative Commons AttributionNonCommercial 3.0 Unported License. To view a copy of this license, visit http://creativecommons.org/licenses/by-nc-nd/3.0/

\section{Executive summary}

Aim

- The authors wished to assess whether atomic force microscopy can be used to probe the elastic properties of human myofibers from live or frozen tissues in situ.

\section{Results}

- This study demonstrates the feasibility of assessing various human muscular dystrophies at the nanometer scale, as based on atomic force microscopy assays of frozen human clinical biopsies.

- Biopsies from dystrophic patients all showed presence of myofibers with abnormally low mechanical resistance to deformation, even for very mild dystrophies that are difficult to diagnose unambiguously from muscle strength or other assays.

- One consulting patient with normal muscle histology and circulating muscle markers but with cardiac arrhythmia also showed soft myofibers, implying occurrence of a muscular dystrophy undetectable by other assays.

\section{Conclusion}

- Myofiber elasticity, described quantitatively by the Young's modulus, may thus be useful in the diagnosis of muscular dystrophies with uncharacterized or otherwise undetectable molecular or cellular defects.

\section{References}

Papers of special note have been highlighted as:

- of interest

-. of considerable interest

1 Petrof BJ, Shrager JB, Stedman HH, Kelly AM, Sweeney HL. Dystrophin protects the sarcolemma from stresses developed during muscle contraction. Proc. Natl Acad. Sci. USA 90(8), 3710-3714 (1993).

2 Blau HM, Webster C, Pavlath GK. Defective myoblasts identified in Duchenne muscular dystrophy. Proc. Natl Acad. Sci. USA 80(15), 4856-4860 (1983).

3 Lisi MT, Cohn RD. Congenital muscular dystrophies: new aspects of an expanding group of disorders. Biochim. Biophys. Acta 1772(2), 159-172 (2007).

4 Guglieri M, Straub V, Bushby K, Lochmuller H. Limb-girdle muscular dystrophies. Curr. Opin. Neurol. 21(5), 576-584 (2008).

5 Cardamone M, Darras BT, Ryan MM. Inherited myopathies and muscular dystrophies. Semin. Neurol. 28(2), 250-259 (2008).

6 Lieber SC, Aubry N, Pain J, Diaz G, Kim SJ, Vatner $S F$. Aging increases stiffness of cardiac myocytes measured by atomic force microscopy nanoindentation. Am. J. Physiol. Heart Circ. Physiol. 287(2), H645-H651 (2004).

-. Atomic force microscopy assay of myocytes reveals age-linked stiffness.
7 Costa KD, Sim AJ, Yin FC. Non-Hertzian approach to analyzing mechanical properties of endothelial cells probed by atomic force microscopy. J. Biomech. Eng. 128(2), 176-184 (2006).

8 Takai E, Costa KD, Shaheen A, Hung CT, Guo XE. Osteoblast elastic modulus measured by atomic force microscopy is substrate dependent. Ann. Biomed. Eng. 33(7), 963-971 (2005).

9 Mathur AB, Collinsworth AM, Reichert WM, Kraus WE, Truskey GA. Endothelial, cardiac muscle and skeletal muscle exhibit different viscous and elastic properties as determined by atomic force microscopy. J. Biomech. 34(12), 1545-1553 (2001).

10 Rotsch C, Radmacher M. Drug-induced changes of cytoskeletal structure and mechanics in fibroblasts: an atomic force microscopy study. Biophys. J. 78(1), 520-535 (2000).

11 Takai E, Landesberg R, Katz RW, Hung CT, Guo XE. Substrate modulation of osteoblast adhesion strength, focal adhesion kinase activation, and responsiveness to mechanical stimuli. Mol. Cell. Biomech. 3(1), 1-12 (2006).

12 Trickey WR, Vail TP, Guilak F. The role of the cytoskeleton in the viscoelastic properties of human articular chondrocytes. J. Orthop. Res. 22(1), 131-139 (2004).

13 Mahaffy RE, Park S, Gerde E, Kas J, Shih CK. Quantitative analysis of the viscoelastic properties of thin regions of fibroblasts using atomic force microscopy. Biophys. J. 86(3), 1777-1793 (2004). 
14 Cross SE, Jin YS, Rao J, Gimzewski JK. Nanomechanical analysis of cells from cancer patients. Nat. Nanotechnol. 2(12), 780-783 (2007).

- Linkage of altered nanomechanical cell stifness to metastatic cancer cells.

15 Faria EC, Ma N, Gazi E et al. Measurement of elastic properties of prostate cancer cells using AFM. Analyst 133(11), 1498-1500 (2008).

16 Lekka M, Gil D, Pogoda K et al. Cancer cell detection in tissue sections using AFM. Arch. Biochem. Biophys. 518(2), 151-156 (2012).

- Detection of cancer cells within tissue sections in situ.

17 Puttini S, Lekka M, Dorchies OM et al. Gene-mediated restoration of normal myofiber elasticity in dystrophic muscles. Mol. Ther. 17(1), 19-25 (2009).

\section{Affiliations}

- Ruthger W van Zwieten

Laboratory of Molecular Biotechnology, Center for Biotechnology UNIL-EPFL \& Institute of Biotechnology, University of Lausanne, Station 6, EPFL, 1015 Lausanne, Switzerland

- Stefania Puttini

Laboratory of Molecular Biotechnology, Center for Biotechnology UNIL-EPFL \& Institute of Biotechnology, University of Lausanne, Station 6, EPFL, 1015 Lausanne, Switzerland

and

Nerve-Muscle Unit, Department of Clinical Neurosciences, Lausanne University Hospital, Lausanne, Switzerland

- Małgorzata Lekka

The Henryk Niewodniczanski Institute of Nuclear Physics, Polish Academy of Sciences, Kraków, Poland

- $\quad$ Guillaume Witz

Laboratory of Molecular Biotechnology, Center for Biotechnology UNIL-EPFL \& Institute of Biotechnology, University of Lausanne, Station 6, EPFL, 1015 Lausanne, Switzerland and

Nerve-Muscle Unit, Department of Clinical Neurosciences, Lausanne University Hospital, Lausanne, Switzerland

- Evelyne Gicquel-Zouida

Généthon \& CNRS UMR8587, Evry, France
-. Linkage of an altered atomic force microscopy profile to lack of dystrophin in mice muscles.

18 Brockington M, Blake DJ, Prandini $\mathrm{P}$ et al. Mutations in the fukutin-related protein gene (FKRP) cause a form of congenital muscular dystrophy with secondary laminin alpha 2 deficiency and abnormal glycosylation of alphadystroglycan. Am. J. Hum. Genet. 69(6), 1198-1209 (2001).

19 Rajakulendran S, Kuntzer T, Dunand M et al. Marked hemiatrophy in carriers of Duchenne muscular dystrophy. Arch. Neurol. 67(4), 497-500 (2010).

20 Jeannet PY, Mittaz L, Dunand M, Lobrinus JA, Bonafe L, Kuntzer T. Autosomal dominant nemaline myopathy: a new phenotype unlinked to previously known genetic loci. Neuromuscul. Disord. 17(1), 6-12 (2007).

- A novel type of difficult-to-diagnose nemalin myopathy disease.

- Isabelle Richard Généthon \& CNRS UMR8587, Evry, France

- Johannes A Lobrinus

Pathology Service, Lausanne University Hospital, Lausanne, Switzerland

- François Chevalley

Department of Surgery, Lausanne University Hospital, Lausanne, Switzerland

- Harald Brune

Laboratory of Nanostructures at Surfaces, Ecole

Polytechnique Fédérale de Lausanne, Lausanne, Switzerland

- Giovanni Dietler

Laboratory of Physics of Living Matter, Ecole Polytechnique Fédérale de Lausanne, Lausanne, Switzerland

\section{- Andrzej Kulik}

Laboratory of Physics of Living Matter, Ecole Polytechnique Fédérale de Lausanne, Lausanne, Switzerland

- Thierry Kuntzer

Nerve-Muscle Unit, Department of Clinical Neurosciences, Lausanne University Hospital, Lausanne, Switzerland

- Nicolas Mermod

Laboratory of Molecular Biotechnology, Center for Biotechnology UNIL-EPFL \& Institute of Biotechnology, University of Lausanne, Station 6, EPFL, 1015 Lausanne, Switzerland 